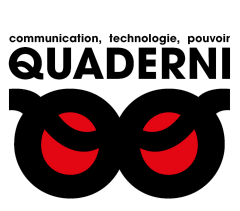

\title{
Quaderni
}

Communication, technologies, pouvoir

\section{Algorithme, management, crise : le triptyque cybernétique du gouvernement de l'exception permanente}

Baptiste Rappin

\section{(2) OpenEdition}

Journals

Édition électronique

URL : https://journals.openedition.org/quaderni/1182

DOI : 10.4000/quaderni. 1182

ISSN : 2105-2956

Éditeur

Les éditions de la Maison des sciences de l'Homme

Édition imprimée

Date de publication : 15 mai 2018

Pagination : 103-114

Référence électronique

Baptiste Rappin, «Algorithme, management, crise : le triptyque cybernétique du gouvernement de l'exception permanente», Quaderni [En ligne], 96 | Printemps 2018, mis en ligne le 15 mai 2020, consulté le 28 septembre 2021. URL : http://journals.openedition.org/quaderni/1182 ; DOI : https:// doi.org/10.4000/quaderni. 1182 


\section{Politique}

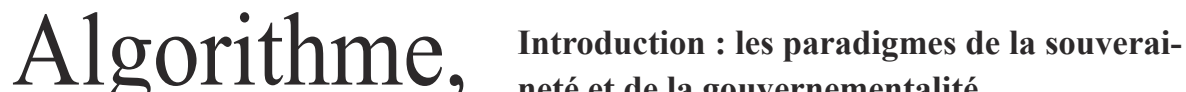 neté et de la gouvernementalité}

\section{management,}

crise : le triptyque cybernétique du gouvernement
de l'exception permanente

Maître de Conférences HDR, IAE de Metz, Université de Lorraine

"République est un droit gouvernement de plusieurs ménages, et de ce qui leur est commun, avec puissance souveraine ", telle est l'entame des Six livres de la République ${ }^{l}$ de Jean Bodin, à qui l'on doit l'introduction du mot « souveraineté » dans son sens moderne. « Droit gouvernement », explique le promoteur de l'absolutisme, car il est nécessaire d'assurer le départ entre la République bien ordonnée, encline à commercer dans la paix, et les troupes de brigands, qui ne sèment que zizanie et ne provoquent que ruines dans les contrées qu'ils traversent. Au souverain, il est en effet donné de créer et de casser les lois, droit fondamental sous lequel se subsument tous les autres : déclarer la guerre et traiter la paix, instituer les officiers, juger en dernier ressort, gracier un condamné, les quatre autres actes régaliens de la souveraineté, découlent en effet de ce « privilège capital » de donner et d'abroger la loi.

C'est tout le brio de Michel Foucault d'avoir montré qu'une telle conception, entièrement solidaire du droit et de ce fait par trop abstraite, était insuffisante pour comprendre les rouages et les dispositifs du pouvoir moderne : «À la vieille mécanique du pouvoir de souveraineté, beaucoup trop de choses échappaient, à la fois par en bas et par en haut, au niveau du détail et au niveau de la masse. C'est pour rattraper le détail qu'une première accommodation a eu lieu : accommodation des mécanismes de pouvoir sur le corps individuel, avec surveillance et dressage - cela a été la discipline. [...] Et puis vous avez ensuite, à la fin du XVIII', une seconde accommodation, sur les phénomènes globaux, sur les phénomènes 
de population, avec les processus biologiques ou bio-sociologiques des masses humaines $\rrbracket^{2}$. D'un côté, le pouvoir disciplinaire contrôle ce qui échappe par nature au souverain, agit sur les individus, plus particulièrement sur les corps, et relève d'une « anatomo-politique » qui soumet les corps à la surveillance dans les prisons, les asiles, les ateliers. De l'autre, tout aussi irréductible au pouvoir souverain qu'à la discipline, la biopolitique s'exerce sur des populations, c'est-à-dire un ensemble d'êtres vivants, «la multiplicité des hommes comme masse globale affectée de processus d'ensemble qui sont propres à la vie $»^{3}$. La démographie et les statistiques, mais aussi la médecine, priment désormais en ce qu'elles prennent en charge le traitement des problèmes de la population, problèmes de vie et de mort : vieillesse, accidents, assurance. Par voie de conséquence, penser la gouvernementalité nécessite de rompre avec le giron juridique de la souveraineté : afin de se détacher de l'image «du pouvoir-loi, du pouvoir-souveraineté que les théoriciens du droit et de l'institution monarchique ont dessinée [...], il faudra bâtir une analytique du pouvoir qui ne prendra plus le droit pour modèle et pour code $»^{4}$.

\section{Du gouvernement de la norme au gouver- nement de l'exception}

Précisons la forme que prend la gouvernementalité chez Foucault : il s'agit d'une gouvernementalité par les normes ${ }^{5}$. Or, il se pourrait que, dans notre société du risque, les situations quasirévolutionnaires, inéluctablement engendrées par le processus de modernisation, se multiplient en prenant «le déguisement de la normalité» selon la très belle expression d'Ulrich Beck ${ }^{6}$. Révo- lution silencieuse, donc, que celle de l'exception : c'est justement tout le mérite d'Agamben que d'avoir introduit ce concept dans le débat contemporain de la philosophie politique.

Là où Foucault faisait état d'une irréductibilité foncière entre les trois modalités d'exercice du pouvoir (souveraineté, pouvoir disciplinaire, biopolitique), le philosophe italien envisage le rapport de la souveraineté à la vie, allant jusqu'à voir dans cette seconde le fondement ultime et secret de la première : «La présente recherche concerne ce point de jonction caché entre le modèle juridico-institutionnel et le modèle biopolitique du pouvoir. L'un des résultats auxquels elle est parvenue est précisément le constat que les deux analyses ne peuvent être séparées, et que l'implication de la vie nue dans la sphère politique constitue le noyau originaire - quoique occulté - du pouvoir souverain. On peut dire en fait que la production d'un corps biopolitique est l'acte originaire du pouvoir souverain »? Agamben retourne alors chez Aristote, qui assure le départ entre le bios, le mode de vie politique, et la zoé, la vie naturelle, cette seconde se trouvant d'emblée exclue de la cité : la vie politique se fonde et se constitue dès son coup d'envoi grec par l'inclusion d'une exclusion, la souveraineté repose dès le départ et constitutivement sur l'exception et la relation de ban. Ce que Foucault pensait être le propre de la séquestration $\mathrm{du} \mathrm{XIX}^{\mathrm{e}}$ siècle, en opposition à la réclusion du XVIII ${ }^{\mathrm{e}}$, «l'inclusion par exclusion $»^{8}$, Agamben l'étend à l'ensemble de la philosophie politique occidentale.

Le philosophe italien s'appuie sur une figure énigmatique que l'on rencontre dans le droit 
romain, celle de l'Homo sacer, cet homme jugé pour crime qu'il est impossible de sacrifier mais dont l'assassin ne sera pas condamné pour homicide : « L'Homo sacer présenterait la figure originaire de la vie prise dans le ban souverain et garderait ainsi la mémoire de l'exclusion originaire à travers laquelle s'est formée la dimension politique. L'espace politique de la souveraineté se serait alors constitué à travers une double exception, telle une excroissance du profane dans le religieux et du religieux dans le profane qui dessine une zone d'indifférence entre le sacrifice et l'homicide. On dira souveraine la sphère dans laquelle on peut tuer sans commettre d'homicide et sans célébrer de sacrifice; et sacrée, c'est-àdire exposée au meurtre et insacrifiable, la vie qui a été capturée dans cette sphère $"{ }^{9}$. Le ban est la prérogative du souverain à l'origine de l'état d'exception comme structure d'inclusion par l'exclusion, le banni se trouvant sous le coup de l'arbitraire souverain tout en étant a-ban-donné par la loi : il se trouve en un seuil où vie et droit se confondent, où intérieur et extérieur entrent en une zone d'indistinction et d'indétermination, en un endroit où il n'existe plus de hors-la-loi, la loi se maintenant dans sa propre privation, s'appliquant dans sa non-application : " la vie nue est ce qui est banni au double sens de ce qui est exclu de la communauté, mis au ban, mais qui est de cette manière mis sous l'enseigne du souverain ${ }^{10}$. La vie nue deviendra, avec l'avènement de la modernité, la forme de vie dominante en fusionnant avec l'espace politique lui-même : bios et zoé entrent dans une zone d'indifférenciation qui explique précisément la montée en puissance du biopouvoir à partir des $\mathrm{XVII}^{\mathrm{e}}$ et $\mathrm{XVIII}^{\mathrm{e}}$ siècles. Mais dire que "la vie nue se libère », c'est affirmer dans le même temps que nos sociétés modernes ont fait de l'exception une règle et que l'état d'exception accède à sa pleine réalisation dans l'espace des camps de concentration. En somme, le philosophe italien énonce une étiologie qui ramène cette crise à l'advenue historique du nazisme et des camps : une thèse provocante, qui possède assurément le mérite de donner à penser, mais éclipse complètement le rôle matriciel de la cybernétique dans la généralisation des pratiques du gouvernement de l'exception.

\section{Au cœur du gouvernement de l'exception permanente : cybernétique, gouvernement et pilotage}

Mais dans quelle direction interprétative se dirige la réception de la pensée d'Agamben ? Elle se concentre quasi-exclusivement, du fait de la réinscription du biopouvoir au sein même de la souveraineté, sur l'identification des figures contemporaines d'Homo Sacer : les immigrés illégaux («sans papiers ») ${ }^{11}$, les demandeurs d'asile $^{12}$, les populations déplacées, les populations colonisées ${ }^{13}$, les prisonniers de Guantanamo $^{14}$, les réfugiés ${ }^{15}$, les victimes de catastrophe naturelle telle que l'ouragan Katrina ${ }^{16}$ ainsi que les destinataires des actions de discrimination positive $^{17}$. Le prisme de la souveraineté influe manifestement sur le choix des objets d'étude qui penchent vers le droit, les relations internationales et la géopolitique. Il nous revient alors de poursuivre cet effort et de montrer que la cybernétique constitue la matrice du gouvernement contemporain de l'exception permanente.

Que la cybernétique se définisse principiellement comme une théorie du gouvernement s'inscrit tout d'abord dans son étymologie : le grec kuber- 
nêtês désigne en effet le pilote du navire, ou le timonier placé sous ses ordres, et kubernêtiké l'art du pilotage ou du gouvernement ${ }^{18}$. Précisons alors que ce dernier terme est issu du latin gobernator, qui renvoie en premier lieu à la personne tenant le gouvernail (gubernaculum) dans ses mains avant de faire signe vers l'homme politique ${ }^{19}$, et n'est autre que la traduction latine du grec kubernêtês. En d'autres termes, cybernétique et gouvernement sont des noms communs strictement synonymes qui tous deux s'enracinent en premier lieu dans le champ sémantique nautique pour ensuite s'étendre au domaine politique de la cité.

Norbert Wiener, dans l'ouvrage de 1948 qui officialisa l'existence de la cybernétique dont l'élaboration était en cours lors des Conférences Macy (1946-1953), donne les raisons précises du choix du terme : «Nous avons décidé de donner à la théorie entière de la commande et de la communication, aussi bien chez l'animal que chez la machine, le nom de cybernétique, formé

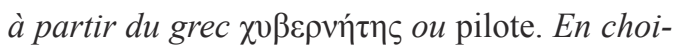
sissant ce terme, nous voulons reconnaître que le premier article significatif sur les mécanismes de rétroaction est un article sur les gouvernails publié par Clerk Maxwell en 1868, et gouvernail

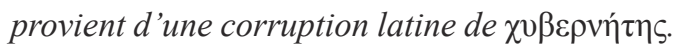
Nous souhaitons aussi rappeler que les appareils de pilotage de navire sont l'une des formes les plus anciennes et les plus perfectionnées de mécanisme de rétroaction $»^{20}$. La cybernétique est indissociable des phénomènes de rétroaction, et cela concerne aussi bien le vaisseau que toute autre entité qui poursuit une direction ou une finalité. Le gouvernement des hommes s'efface derrière le mécanisme du feedback dont l'extension généralisée aboutira à la gouvernance des processus. Le savoir que la cybernétique produit concerne plus précisément 1 ' « organisation », terme générique qui désigne toute boucle de rétroaction qui tend à résister à l'entropie, ce mouvement général qui conduit lentement mais sûrement l'univers à une indistinction générale. Observons alors les mots que Norbert Wiener ne cesse d'utiliser pour souligner la précarité de l'organisation; pour lui, elle est une « enclave $»^{21}$, une $"$ poche $»^{22}$, un " processus local $»^{23}$, une " île temporaire $»^{24}$. Échappant à la seconde loi de la thermodynamique, l'organisation est pleinement une exception : son existence est hautement improbable, ce qui en fait une exception statistique ; et elle s'impose provisoirement mais réellement à l'entropie, ce qui en fait une exception ontologique. Alors, si nous convenons avec le mathématicien de la multiplication des organisations (naturelles, vivantes, artificielles) qui marquent l'époque planétaire, si nous convenons qu' « en notre monde l'entropie ne s'accroît pas, alors que l'organisation et son corollaire l'information se développent ${ }^{25}$, il nous faut bien conclure, en reprenant l'expression de Walter Benjamin, que «l'état d'exception dans lequel nous vivons est devenu la règle $»^{26}$. Observons alors son déploiement: dans le gouvernement des données, dans le gouvernement managérial, dans le gouvernement critique.

\section{Le gouvernement des données}

Le gouvernement de l'exception permanente s'observe tout d'abord dans le projet de digitalisation de la vie et du monde. La cybernétique, par l'anthropologie naturaliste et informationnelle qu'elle promeut, contient dès son projet initial la convergence des technologies que l'on 
désigne aujourd'hui sous l'acronyme NBIC (Nanotechnologies, Biotechnologies, Informatique, sciences Cognitives). C'est dans ce cadre plus général que doit être compris le gouvernement des données, encore appelé Big Data en raison de l'enregistrement et du traitement massifs et même systématiques des informations opérés par des algorithmes sophistiqués dont les capteurs nous suivent de trace en trace : cookies, satellites, téléphones intelligents, cartes bancaires, administrations, etc., fournissent des éléments permettant de dresser et d'actualiser notre profil comportemental en temps réel. Ce mouvement de numérisation semble illimité tant les systèmes informatiques contemporains possèdent des capacités de stockage inimaginables, et ouvre la voie au data mining, c'est-à-dire à des techniques de modélisation qui cherchent à mettre au jour les structures enfouies dans la masse de données.

Or, la captation, le traitement, l'analyse et le maniement de ces données remettent profondément en cause le mode statistique de gouvernementalité qui considère toute variation comme un défaut à gommer ou un fossé à combler : considérant par exemple l'homme moyen d'Adolphe Quételet, l'on mettra en œuvre des mesures de réduction des écarts visant à ramener les brebis égarées dans le troupeau de la norme considérée comme bonne. Mais la gouvernementalité algorithmique s'avère " incommensurable avec les différents arts de gouverner décrits par Foucault sous les traits du pastorat, de la raison d'État, de la police ou encore du libéralisme $\${ }^{27}$. À quoi tient cette impossibilité de la comparaison ? C'est que le gouvernement des données, comme l'écrivent Antoinette Rouvroy et Thomas Berns, désigne « un certain type de rationalité (a) normative ou (a)politique reposant sur la récolte, l'agrégation et l'analyse automatisée de données en quantité massive de manière à modéliser, anticiper et affecter par avance les comportements possibles $»^{28}$. Les auteurs poursuivent en soulignant les déplacements opérés entre le gouvernement statistique et la gouvernance algorithmique : l'apparente individualisation d'une part, puis la participation voire l'accord et même l'adhésion de l'individu à la constitution de son profil d'autre part. Il est vrai que la plupart d'entre nous n'hésite guère, alors qu'elle navigue sur la toile, à remplir les cases du profil client à la moindre commande, ni à activer la géolocalisation lorsque celle-ci est suggérée par un site ou une application. Mais plus encore, la gouvernementalité algorithmique s'actualise en temps réel car elle ingurgite immédiatement toute nouvelle donnée, la mettant en correspondance avec d'anciennes informations pour proposer de nouvelles corrélations, plastiques, mobiles, et modelables à souhait, bref des exceptions permanentes : dans un tel modèle, il n'est en effet plus fait référence à une instance surplombante ; au contraire, la norme est immanente au calcul de telle sorte qu'elle évolue en même temps que lui et ne laisse plus aucune place à l'écart qui se trouve automatiquement et instantanément avalé et intégré par l'algorithme : "Indifférente aux causes des phénomènes, cette rationalité s'ancre dans l'observation purement statistique de corrélations (indépendantes de toute logique) entre données recueillies d'une manière absolument non sélective dans une variété de contextes hétérogènes les uns aux autres $»^{29}$. La norme en vient à épuiser la totalité du réel, et aucun possible ne saurait résister à son actualisation. Et puisque le gouvernement des données s'appuie sur des corrélations qu'elle 
ne relie à aucune origine ni ne contextualise, il tend à dresser des profils et des modèles, des signifiants, sans aucun lien avec le réel ou avec une signification, mais qui demandent à être actualisés : il possède de ce point de vue une indéniable dimension performative, car, sous sa houlette, les individus se voient incités à devenir non pas ce qu'ils sont, mais ce qu'ils doivent à chaque instant être, et même ce qu'ils pourraient être : «Le gouvernement statistique vise non plus à maîtriser l'actuel, à dompter la sauvagerie des faits, mais à structurer le possible $»^{30}$.

\section{Le gouvernement managérial}

Il est difficile de constater la présence du mot « cybernétique » dans le champ du management. Mais le succès du terme « pilotage », qui pointe directement vers l'étymologie de la cybernétique, nous met sur la bonne piste : « pilotage de la valeur », « pilotage des coûts », « pilotage de la performance », « pilotage des organisations », « pilotage par les tableaux de bord », « pilotage du changement », « pilotage social », « pilotage des ressources humaines », «pilotage des systèmes d'information », « pilotage des services publics », etc., tous ces titres et sous-titres témoignent de l'ancrage du management contemporain dans la matrice cybernétique.

En outre, mes travaux généalogiques ${ }^{31}$ mettent en exergue le lien direct qui unit le management de l'après-guerre à la cybernétique ; en effet, les principales disciplines (le management stratégique, le contrôle de gestion, les systèmes d'information, la gestion des ressources humaines, le management des connaissances, pour ne citer qu'elles) se greffent directement sur le schéma de la boucle de rétroaction et conçoivent l'entreprise, et même toute entité collective, comme une organisation qui met en place des systèmes d'apprentissage (des «feedbacks positifs ») destinés à générer un fonctionnement efficace par la maîtrise des circuits d'information. C'est par ce mécanisme que se joue l'adaptation et l'ajustement perpétuels à l'environnement.

On pourrait alors croire que cette conception cybernétique du gouvernement ne concerne que les seules entreprises au sein desquelles, il est vrai, le management a trouvé un fertile terreau d'application et de développement ; cependant, loin de se cantonner à cette seule sphère, le pilotage fournit à la gouvernementalité son paradigme général. Il revint plus particulièrement au politiste Karl Deutsch d'avoir perçu les implications politiques de la révolution informationnelle, et d'avoir proposé une théorie du politique qui assimile la société à un système nerveux. S'appuyant directement sur les propositions de la cybernétique, il conçoit le gouvernement comme « un processus de pilotage » qui procède du jeu des comportements finalisés et des boucles négatives ou amplificatrices de rétroaction ${ }^{32}$. Cet impérialisme et cette extension de la cybernétique, qui avale la politique dans son giron, sont rendus possibles par l'attribution de la catégorie d' " organisation » à toute activité collective : «The viewpoint of cybernetics suggests that all organizations are alike in certain fundamental characteristics and that every organization is held together by communication. [...] Finally, cybernetics suggests that steering or governing is one of the most interesting and significant processes in the world, and that a study of steering in self-steering machines, in biological organisms, 
in human minds, and in societies will increase our understanding of problems in all these fields $»^{33}$.

Mais le propos devient d'autant plus intéressant que Karl Deutsch ne recule pas devant les conséquences politiques de son ancrage cybernétique : il s'agit bien au fond de substituer la gouvernementalité à la souveraineté. Qu'entend en effet le politologue par " autonomie»? Non plus la capacité politique d'un peuple à se donner ses propres lois, mais l'aptitude technique d'un système à changer ses finalités grâce à sa mémoire ainsi qu'aux interactions qu'il noue avec son environnement $^{34}:$ l'autonomie n'est rien d'autre, au total, que la maitrise de l'information. Ce projet allait d'ailleurs trouver un terrain d'expérimentation dans le Chili du socialiste Salvador Allende; en 1971, Fernando Flores, en charge du management auprès du nouveau président, fit en effet appel au cybernéticien Stafford Beer, dont la réputation était alors internationale, pour mettre en application les principes du pilotage à l'échelon non plus d'une entreprise mais d'une nation. C'est ainsi que vit le jour le projet Cybersyn, un système d'information décentralisé, préfigurant en ce sens les réseaux contemporains, destiné à réguler en temps réel l'économie chilienne à partir des données captées au niveau local ${ }^{35}$.

\section{Le gouvernement critique}

Les auteurs de la revue Tiqqun mettent en lumière le passage de l'hypothèse libérale, dont le mythe fondateur serait celui de la Fable des Abeilles de Bernard de Mandeville ${ }^{36}$, à l'hypothèse cybernétique qui devient, à partir de la Seconde Guerre mondiale, le paradigme de gouvernement dominant : «Le libéralismen'est plus désormais qu'une justification rémanente, l'alibi du crime quotidien perpétré par la cybernétique $\|^{37}$. Ce projet d'une nouvelle technologie de gouvernement quitte le sol de la liberté et de l'individualisme pour gagner la région du contrôle et du pilotage : «À l'opposé de cette l'hypothèse libérale, l'hypothèse cybernétique propose de concevoir les comportements biologiques, physiques, sociaux comme intégralement programmés et re-programmables. Plus précisément, elle se représente chaque comportement comme "piloté» en dernière instance par la nécessité de survie d'un "système" qui le rend possible et auquel il doit contribuer $»^{38}$. Le rapport de force entre capitalisme et cybernétique s'est inversé, et «l'information est devenue la richesse à extraire et à accumuler, transformant le capitalisme en auxiliaire de la cybernétique $\aleph^{39}$. Le régime de la fluidification supplante celui de l'accumulation. Certes le terme disparut très vite, mais « de 1954 au temps présent, le projet que s'était assigné le groupe cybernétique n'a cessé d'être remis sur le chantier sous des appellations à chaque fois différentes $»^{40}$. Que l'on évoque ainsi l'intelligence artificielle et la puissance des algorithmes, que l'on fasse référence aux sciences cognitives ou aux puces RFID, que l'on s'attache encore au continent du management et de la nouvelle économie, toutes ces modalités du gouvernement contemporain pointent vers une même matrice : la cybernétique, c'est-à-dire le pilotage et le contrôle de l'information finalisée.

Or, poursuivent les auteurs de Tiqqun, la cybernétique " est une pensée de l'équilibre née dans un contexte de crise $»^{41}$ : au sortir de la Seconde Guerre mondiale, dans le souvenir de la première, gardant en tête le traumatisme des 
bombes nucléaires lâchées sur le Japon, effarés par la découverte progressive de l'horreur des camps de concentration et d'extermination, les scientifiques américains se mirent en quête d'une science capable de ramener l'ordre et la certitude dans le chaos ; plus encore, ils cherchèrent à tirer l'ordre du désordre, la certitude de l'incertitude, de telle sorte que la crise devient même l'occasion idoine d'instaurer de nouveaux mécanismes de régulation. De ce point de vue, l'état d'urgence devint l'instrument privilégié du gouvernement : « La cybernétique vise par conséquent à inquiéter et à contrôler dans le même mouvement. Elle est fondée sur la terreur qui est facteur d'évolution - de croissance économique, de progrès moral - car elle fournit l'occasion d'une production d'informations. L'état d'urgence, qui est le propre des crises, est ce qui permet à l'autorégulation d'être relancée, de s'auto-entretenir comme mouvement perpétuel $\gg^{42}$.

Ainsi s'opère-t-il un saut décisif qui mène de la conjoncture historique à la structure ontologicopolitique : alors que la crise est initialement à la fois le contexte dans lequel évoluent les scientifiques des Conférences Macy et le problème qu'ils se proposent de résoudre, elle devient une catégorie centrale, tout ensemble métaphysique et stratégique : métaphysique, car elle est conceptualisée comme la condition de possibilité de nouvelles régulations et l'origine d'un nouvel ordre; stratégique, car elle devient un opérateur et un levier qu'il est possible de manier artificiellement. Nous pouvons toutefois formuler cette thèse de façon plus rigoureuse ; car ce n'est pas à proprement parler un « ordre », avec ses connotations de stabilité, de régularité et de succession logique, qui émane du désordre : il s'agit bien plutôt d'un désordre organisé, c'est-à-dire d'une organisation dans laquelle la déstabilisation tout autant que la nécessité de l'adaptation sont permanentes.

On ne peut alors qu'être frappé par la proximité qui s'établit entre le projet cybernétique, celui d'un gouvernement de et par l'exception, et la «stratégie du choc » que Naomi Klein présente de façon documentée. Quelle est la thèse en question? "Voici donc comment fonctionne la stratégie du choc: le désastre déclencheur - le coup d'État, l'attentat terroriste, l'effondrement des marchés, la guerre, le tsunami, l'ouragan - plonge la population dans un état de choc collectif. Le sifflement des bombes, les échos de la terreur et les vents rugissants "assouplissent» les sociétés, un peu comme la musique tonitruante et les coups dans les prisons où se pratique la torture. À l'instar du prisonnier terrorisé qui donne le nom de ses camarades et renie sa foi, les sociétés en état de choc abandonnent des droits que, dans d'autres circonstances, elles auraient défendus jalousement $»^{43}$. Telle est la puissante analogie qui traverse l'ouvrage de Naomi Klein : le gouvernement par la crise s'applique aussi bien aux individus qu'aux sociétés. Et de même que le prisonnier, las des divers et multiples traumatismes que ses tortionnaires lui infligent, finit par céder, de même les sociétés, prises de panique lorsque les repères traditionnels et coutumiers disparaissent, sont prêtes à bien des concessions, et notamment à la mise en place de l'arsenal néolibéral. Et la journaliste d'étayer et d'affermir ce parallèle en comparant les trajectoires des deux figures du docteur Ewen Cameron, le directeur de l'Institut Allan Memorial de l'Université McGill à Montréal, et de l'économiste Milton Friedman, responsable du fameux département des Sciences 
Économiques de l'Université de Chicago.

\section{Conclusion : l'organisation et le gouvernement de l'exception permanente}

Edgar Morin livre à son lecteur le secret de la lutte contre l'entropie :

« La seule possibilité de lutter contre la désintégration est :

- d'intégrer et utiliser le plus possible les antagonismes de façon organisationnelle ;

- de renouveler énergie et organisation en les puisant dans l'environnement (système ouvert) ; - de pouvoir s'automultiplier de façon à ce que le taux de reproduction dépasse le taux de dégradation ;

- d'être capable de s'autoréorganiser, de s'autodéfendre $»^{44}$.

Une organisation survit si elle s'ouvre à son environnement afin d'introduire en son sein de la variété, et si elle met en œuvre des processus de régulation et de contrôle qui permettent d'orienter les actions dans le sens de la finalité définie. Mais à quelles conditions l'énergie et l'information peuvent-elles circuler non seulement de l'environnement vers l'organisation, et réciproquement, mais également au sein même de l'organisation? C'est ici qu'intervient le concept de « zone différentielle» introduit par Yves Stourdzé : «Les organisations s'inscrivent à l'intérieur de zones. Par le jeu des différences entre celles-ci, la circulation énergétique devient possible. [...] Instaurer des déséquilibres dans des zones, entre les zones, telle est la politique de l'organisation à la recherche d'énergie extérieure $\gg^{45}$.
Dans un univers en état d'équilibre thermique, dans lequel l'entropie aurait vaincu toutes formes de vie, aucun événement, en d'autres termes aucun échange d'information, ne saurait se produire car il y manque cette dénivellation qui permet de distinguer un « avant » et un « après », un point de départ et un point d'arrivée. L'écart se révèle ainsi la condition de possibilité de la circulation et $\mathrm{du}$ fonctionnement. C'est pourquoi le maintien de l'organisation passe par la création et l'entretien de la crise, car seule la rupture engendre la différenciation des niveaux et ouvre alors le chemin au transit de l'information. Et, de ce point de vue, il faut absolument garder en ligne de mire que la crise ne peut être séparée du contrôle, les deux formant un couple indissociable : sans déséquilibre, l'information se trouve dans l'incapacité de circuler et ne parvient pas à l'organisation ; sans contrôle, l'information se révèle être une force corrosive et entraîne l'organisation vers la déstructuration. Algorithme, management, crise sont alors les trois leviers du gouvernement de l'exception permanente. 
$\mathrm{N} \cdot \mathrm{O} \cdot \mathrm{T} \cdot \mathrm{E} \cdot \mathrm{S}$

1. Jean Bodin, Les six livres de la République, Paris, Librairie générale française, «Classiques de la philosophie », 1993, p. 57.

2. Michel Foucault, «Il faut défendre la société ». Cours au Collège de France, 1976, Paris, Gallimard Seuil, « Hautes Études », 1997, p. 222-223.

3. Ibid., p. 216.

4. Ibid., p. 118-119.

5. Pierre Macherey, De Canguilhem à Foucault. La force des normes, Paris, La Fabrique éditions, 2009.

6. Ulrich Beck, La société du risque. Sur la voie d'une autre modernité, trad. Laure Bernardi, Paris, Flammarion, « Champs », 2001, p. 143.

7. Giorgio Agamben, Homo Sacer. Le pouvoir souverain et la vie nue, trad. Marilène Raiola, Paris, Seuil, «Ordre philosophique », 1997, p. 14.

8. Michel Foucault, «La vérité et les formes juridiques », 1974, repris dans Dits et écrits. 1954-1975, Paris, Éditions Gallimard, « Quarto », 2001, p. 1482.

9. Giorgio Agamben, Homo Sacer. Le pouvoir souverain et la vie nue, op. cit., p. 93.

10. Katia Genel, « Le biopouvoir chez Foucault et Agamben », Methodos, 2004, n 4, p. 7.

11. Willem Schinkel, « Illegal Aliens and the State, or: Bare Bodies vs the Zombies ", International Sociology, 2009, n 24, p. 779-806.

12. Jon Stratton, «Zombie trouble: Zombie texts, bare life and displaced people », European Journal of Cultural Studies, 2011, n 14, p. 265-281.

13. Susan Diane Brophy, «Lawless Sovereignty: Challenging the State of Exception », Social \& Legal Studies, 2009, n 18, p. 199-220.

14. Andrew W. Neal, « Foucault in Guantanamo : Towards an Archaeology of the Exception », Security Dialogue, 2006, $\mathrm{n}^{\circ} 37$, p. 31-46
15. Jenny Edkins, « Sovereign Power, Zones of Indistinction, and the Camp », Alternatives : Global, Local, Political, 2000, $\mathrm{n}^{\circ}$ 25, p. 3-25.

16. Ben Chappell, "Rehearsals of The Sovereign : States of Exception and Threat Governmentality », Cultural Dynamics, 2006, n 18, p. 313-334.

17. Daniel Sabbagh, « La discrimination positive : une "politique de l'exception" ? ", Tracés. Revue de Sciences Humaines, 2011, n 20, p. 97-106.

18. Anatole Bailly, Dictionnaire Grec Français, entrées « Hachette, 2000, p. 1145-1146.

19. Félix Gaffiot, Dictionnaire Latin Français, entrées « gubernaculum » et « gubernator », Paris, Hachette, 2000, p. 732.

20. Norbert Wiener, La cybernétique. Information et régulation dans le vivant et la machine, trad. Ronan Le Roux, Robert Vallée et Nicole Vallée-Lévi, Paris, Éditions du Seuil, « Sources du savoir », 2014, p. 70-71.

21. Norbert Wiener, The Human Use of Human Beings. Cybernetics and Society, Boston, Da Capo Press, « Science/Sociology », 1988, p. 31.

22. Ibid., p. 32.

23. Loc. cit.

24. Ibid., p. 36, p. 95.

25. Ibid., p. 31.

26. Walter Benjamin, «Sur le concept d'histoire », VIII, in Euvres III, trad. Maurice de Gandillac, Rainer Rochlitz et Pierre Rusch, Paris, Éditions Gallimard, « folio essais », 2000, p. 433.

27. Antoinette Rouvroy et Thomas Berns, « Le nouveau pouvoir statistique. Ou quand le contrôle s'exerce sur un réel normé, docile et sans événement car constitué de corps "numériques"... », Multitudes, 2010, n 40, p. 89.

28. Antoinette Rouvroy et Thomas Berns, « Gouvernementalité algorithmique et perspectives d'émanci- 
pation. Le disparate comme condition d'individuation par la relation ?», Réseaux, 2013, n 177, p. 173. 29. Antoinette Rouvroy et Thomas Berns, « Le nouveau pouvoir statistique. Ou quand le contrôle s'exerce sur un réel normé, docile et sans événement car constitué de corps "numériques"... », art. cité, p. 91 .

30. Ibid., p. 93.

31. Baptiste Rappin, $A u$ fondement du Management. Théologie de l'Organisation, Volume 1, Nice, Éditions Ovadia, "Chemins de pensée », 2014 ; Heidegger et la question du Management. Cybernétique, information et organisation à l'époque de la planétarisation, Nice, Éditions Ovadia, «Chemins de pensée », 2015 ; De l'exception permanente. Théologie de l'Organisation, Volume 2, Nice, Éditions Ovadia, « Temps présents », 2018.

32. Karl W. Deutsch, The Nerves of Government. Models of Political Communication and Control, New York, The Free Press, 1966, p. 182 sq.

33. Ibid., p. 77-78.

34. Ibid., p. 128.

35. Eden Medina, Cybernetics Revolutionaries. Technology and Politics in Allende's Chile, Cambridge, The MIT Press, 2011.

36. Robert-Dany Dufour, Pléonexie [dict. : «Vouloir posséder toujours plus], Lormont, Le bord de l'eau, « La bibliothèque du Mauss », 2015.

37. Tiqqun, Organe de liaison au sein du Parti Imaginaire. Zone d'Opacité Offensive, Paris, Les Belles Lettres, 2001, p. 41.

38. Ibid., p. 42.

39. Ibid., p. 51.

40. Jean-Pierre Dupuy, Aux origines des sciences cognitives, Paris, Éditions La Découverte, « Sciences humaines et sociales », 1999, p. 7.

41. Tiqqun, Organe de liaison au sein du Parti Imaginaire. Zone d'Opacité Offensive, op.cit., p. 42.
42. Ibid., p. 52.

43. Naomi Klein, La stratégie du choc. La montée d'un capitalisme $d u$ désastre, trad. Lori StMartin et Paul Gagné, Montréal, Leméac / Actes Sud, « Babel », 2008, p. 31.

44. Edgar Morin, Pour une crisologie, Paris, L'Herne, « Carnets », 2016, p. 32.

45. Yves Stourdzé, Organisation, anti-organisation, Paris, Sens\&Tonka, 2015, p. 25-26. 


\title{
$R \cdot E ́ \cdot S \cdot U \cdot M \cdot E ́$
}

Michel Foucault a montré comment la compréhension du pouvoir et de ses mécanismes ne pouvait se limiter à la seule souveraineté ; c'est la raison pour laquelle il introduisit la notion de biopouvoir, dans sa double dimension de discipline et de biopolitique. Agamben s'inscrit dans les pas du philosophe français mais opère un retournement : il montre en effet que la gouvernementalité repose moins sur la norme que sur l'exception. Notre article vise à poursuivre cet effort en établissant que la cybernétique constitue la matrice du gouvernement contemporain de l'exception permanente. Sont alors passées en revue les trois modalités de ce nouveau pouvoir : le gouvernement algorithmique, le gouvernement managérial, le gouvernement critique.

\begin{abstract}
Michel Foucault showed that the understanding of power and of its mechanisms cannot be restricted to sovereignty; that's why he introduced the notion of biopower, in its double dimension of discipline and biopolitcs. Agamben continues the French philosopher's work, but proceeds to a turnaround: indeed, he establishes that governmentality is less based on norms than on exception. Our article aims at completing this effort by showing that cybernetics constitutes the matrix of contemporary government of permanent exception. We then go through the three forms of that new power: algorithmic government, managerial government and critical government.
\end{abstract}

